\title{
Intermédialités
}

Histoire et théorie des arts, des lettres et des techniques

Intermediality

History and Theory of the Arts, Literature and Technologies

\section{Des vertus heuristiques de l’intermédialité}

\section{François Jost}

Numéro 6, automne 2005

URI : https://id.erudit.org/iderudit/1005509ar

DOI : https://doi.org/10.7202/1005509ar

Aller au sommaire du numéro

Éditeur(s)

Centre de recherche sur l'intermédialité

ISSN

1705-8546 (imprimé)

1920-3136 (numérique)

Découvrir la revue

Citer cet article

Jost, F. (2005). Des vertus heuristiques de l'intermédialité. Intermédialités /

Intermediality, (6), 109-119. https://doi.org/10.7202/1005509ar
Résumé de l'article

Que désigne le concept d'intermédialité et quelle est sa vertu heuristique pour la théorie? En procédant à un petit essai d'" ego-histoire ", cet article propose différentes réponses à cette question. Une chose frappe d'abord : si l'intermédialité a souvent été une idéologie d'artiste nécessaire à la création (voir Eisenstein), elle ne joue presque aucun rôle dans la première sémiologie du cinéma et dans la narratologie littéraire. Elle est « une question non questionnée ", comme aurait dit Bachelard. La première vertu heuristique du concept est celle de la narratologie comparée, qui consiste à faire varier en extension et en compréhension des concepts peu ou prou universels. Elle procède par allers-retours entre mediums. Ces résultats restent fragiles tant qu'ils n'ont pas été confrontés à l'épreuve de la mise en contexte médiatique. En s'appuyant sur les exemples du début du cinéma et du succès mondial d'émissions du type Big Brother, on montre que le concept d'intermédialité n’a d'efficacité réelle que lorsqu'il établit des passerelles entre des médias différents pour mieux construire une relation intelligible entre des causes et des effets.
Ce document est protégé par la loi sur le droit d'auteur. L'utilisation des services d’Érudit (y compris la reproduction) est assujettie à sa politique d'utilisation que vous pouvez consulter en ligne.

https://apropos.erudit.org/fr/usagers/politique-dutilisation/ 


\title{
Des vertus heuristiques de l'intermédialité
}

\author{
FrançOIS JOST
}

uel point de vue adopter pour faire la généalogie d'un concept? Celui de

l'archéologue, qui fouille dans un passé qu'il n'a pas vécu et dont, à partir de quelques traces éparses, il reconstitue tant bien que mal la construction? Celui de l'historien qui se plonge dans les archives et transforme la succession en causalité? Voilà le genre de questions que pose le concept d'intermédialité. Mais comment feindrais-je ces regards surplombants de l'archéologue ou de l'historien, moi qui, peu ou prou, ai été un acteur de cette intrusion progressive de l'intermédialité dans la recherche? Et si, pour une fois, je faisais le choix, qui en vaut bien d'autres, de ce que Le Goff a appelé l'ego-histoire'? biographie, qui se pencherait avec complaisance sur les détours vécus qui m’ont amené à entreprendre telle ou telle recherche, mais bien de cette tentative de mise à distance d'un parcours théorique dans lequel la posture intermédiaire a toujours été privilégiée (deux de mes livres recourent à la conjonction « entre² » et d'autres proposent des mots-valises destinés à qualifier un type d'intermédialité: picto-roman, picto-film ou film-opéra).

1. Voir Pierre Nora (dir.), Essais d'ego-histoire, Paris, Éditions Gallimard, coll. «Bibliothèque des histoires », 1987, dans lequel Pierre Nora a demandé à des historiens français connus de retracer leur propre cheminement professionnel et de l'expliquer en rapport avec l'histoire de la société dans laquelle ils vivaient.

2. François Jost, «Le film-opéra», Critique, n 336 , mai 1975, p. 544-551, repris dans Obliques, $\mathrm{n}^{\mathrm{os}} 16-17,1978$, p. 239-246; "Le picto-roman ", Revue d'esthétique, $\mathrm{n}^{\circ} 4$, 1976, p. 58-73; «Le picto-film», dans Raymond Bellour (dir.), Cinéma et peinture, Paris, Presses universitaires de France, coll. «Écritures et arts contemporains », 1990, p. 109122; L'oil-caméra. Entre film et roman, Lyon, Presses universitaires de Lyon, 1987, 1989 ( ${ }^{\mathrm{e}}$ éd.) ; La télévision du quotidien. Entre réalité et fiction, Bruxelles, De Boeck-INA, 2001, 2004 ( 2 éd.). 
Puisque ce retour fait partie de la démarche épistémologique que j’appelle de mes vœux depuis 10 ans, je le ferai avec une seule question en tête: quels modèles de l'intermédialité sont supposés par les recherches comparées sur les documents audiovisuels et quel rôle heuristique jouent ces concepts dans les théories qui les mobilisent?

\section{LES PREUVES D'ARTISTES}

L'intermédialité inaugure-t-elle un nouveau paradigme théorique ou est-elle d'abord une idéologie d'artiste? Plutôt que de disserter sur ce beau sujet d'examen, dans la perspective historique qui est la mienne, je partirai de la façon dont cette question se pose à moi à la fin des années 1960, au moment où j'entre en théorie. D'un côté, il y a des œuvres, vers lesquelles se tournent les regards de ceux qui revendiquent la modernité; de l'autre, de jeunes théoriciens, souvent les mêmes que les précédents, affirmant la nécessité d'une nouvelle critique. Pour moi, qui, soit dit en passant, suis plus jeune que ces jeunes, qui ont pour nom Genette, Todorov, Barthes, etc., trois œuvres prouvent que la modernité est liée à la revendication de l'intermédialité et provoquent chez moi l'envie d'écrire: Ulysses (1922), de James Joyce, dont le chapitre XI, dit «Les sirènes », est construit sur le modèle d'une fuga per canonem; Aleksandr Nevsky (1938), dans lequel Sergei Eisenstein prétend établir une correspondance entre la montée de ligne mélodique et la ligne ascendante des casques dans l'image, au cours de la fameuse bataille sur glace. Et, enfin, Le jeu avec le feu (1974), d'Alain RobbeGrillet, qui, selon moi, structure son récit en fonction d'une nécessité musicale et non d'une «logique» diégétique.

Ces trois tentatives de marier la musique, par essence non narrative, avec les arts du récit se heurtent évidemment à bien des difficultés:

- l'entreprise d'Eisenstein requiert de faire sienne l'hypothèse cratylique et de postuler la possibilité d'une synesthésie. Peut-on admettre, comme il le fait, que, selon une sorte de correspondance baudelairienne, la perception d'une ligne mélodique équivaille à la perception d'une ligne graphique’?

- la difficulté du parti pris esthétique de Joyce est autre: si le texte est conçu sur le modèle d'un thème musical qui est imité et poursuivi, comment déterminer ce thème, qui est la base de la structure de la fugue, et qu'entendre

3. Voir Sergei Eisenstein, «Le fond, la forme et la pratique» [1940], dans Le film: sa forme, son sens, trad. Armand Panigel, Paris, Christian Bourgois Éditeur, 1976 [1949], p. 317-343. 
par son imitation s'il s'agit de mots, et non plus de textes? En tout état de cause, quoi qu'il en soit des unités pertinentes construites par le lecteur et quoi qu'il en soit des possibilités de faire fonctionner ce modèle, la recherche de ces unités mobilisera chez le lecteur des mécanismes cognitifs bien différents de l'appréhension perceptive mobilisée par le mélomane.

- l'hypothèse du film-opéra se heurte à un nouvel obstacle: celui de la représentation. Si, comme dans le texte littéraire, la recherche des unités a la complexité que je viens d'évoquer, elle se complexifie encore du fait qu'il faut aller chercher sous le montré, en quelque sorte, et sous le narratif, un motif dont les variations feront une structure non narrative et non représentative (par exemple, dans le Jeu avec le feu, «le dessin formé par ces données visuelles et sonores de la structure narrative ["chienne"-fouet-train] tisse un motif entre deux personnages $\left.{ }^{\star}\right)$. Quand nous parlions d'esthétique, le mot «impasse » revenait souvent dans la bouche de Robbe-Grillet pour qualifier la voie la plus personnelle empruntée par un artiste. Et c'est ce mot qui me vient à l'esprit aujourd'hui pour caractériser ces trois essais d'intermédialité qui se moquent bien des diktats de la théorie. En même temps qu'ils se heurtent au problème de la preuve, ils le nient. Ils visent moins à la justesse qu'à la mise en crise des codes de la représentation ou du récit couramment admis par un art. Ils donnent donc l'image d'une intermédialité militante, dont la preuve est seulement pragmatique : elle permet de faire. Le paradoxe de cette intermédialité militante, c'est que, en même temps qu'elle va puiser son inspiration en dehors du champ sémiotique propre, elle s'affirme comme une recherche de la spécificité, puisqu'il s'agit, au fond, d'éprouver les limites de chaque art, de chaque pratique, l'une par l'autre.

\section{L'ÉPREUVE DES LIMITES}

\section{A. L'intermédialité comme tache aveugle}

Les théories littéraires et cinématographiques des années 1960, encore très vivaces dans les années 1970, sont fort mal préparées à penser ces «impasses » d'artistes, dans la mesure où leur cadre les cantonne à l'immanence du texte. On peut s'enfoncer dans le texte jusqu'au granulaire, comme le fait la théorie des «générateurs » de Jean Ricardou, qui décompose quelques mots en anagrammes et autres paragrammes, mais sans l'inclure dans un ensemble

4. François Jost, «Le film-opéra », p. $55^{8}$. 
plus vaste5. D'où le rejet d'une extension homogénéisante, comme la figure de l'auteur, et l'évitement de toute extension hétérogène.

En outre, ces mêmes théories pensent plutôt le classicisme (la sémiologie metzienne du cinéma) ou des configurations possibles du récit (la narratologie de Gérard Genette), deux corpus, si l'on peut dire, qui ne nécessitent nullement de faire entrer en ligne de compte l'intermédialité. La sémiologie du cinéma, par exemple, se caractérise par deux traits:

- son caractère centripète, qui la pousse à éliminer tout ce qui est commun à plusieurs médias. Ainsi Metz englobe-t-il d’abord toutes les images, pour les différencier ensuite, un peu comme on sépare le blanc du jaune d'œuf, par exclusions successives, en fonction du pouvoir plus ou moins englobant des codes à manifestations multiples mais non universelles: iconicité, mise en séquence (cinéma, dessins animés, bande dessinée, fresque, télévision, photo-roman), duplication mécanique et images en mouvement (cinéma, télévision, dessins animés), le critère de la duplication mécanique séparant, en dernière instance, le dessin animé du cinéma et de la télévision.

- cette réduction du médium ou du «langage», comme dit Metz, à la spécificité codique, qui a comme conséquence logique l'éviction de la question de l'intermédialité, entendue cette fois comme relation entre médias : si tout se réduit a un assemblage de codes, peu importent les différences de médiatisation. Ainsi, affirme-t-il, «le cinéma et la télévision, au fond, ne sont rien d'autres que des langages voisins, mais qui poussent le voisinage beaucoup plus loin que ne le font d'ordinaire les langages entre eux [...]. Aussi peut-on les traiter tous deux comme s'ils formaient un langage unique ${ }^{6}$.»

\section{B. L'intermédialité non questionnée}

C'est en réponse à cette situation - et parce qu'il m'importe de penser les impasses d'artistes - que l'intermédialité, d'abord conçue comme relation entre médiums (c'est le mot que j'emploie à l'époque, plutôt que «langage »), va me permettre d'éprouver les limites des théories alors constituées. Tache aveugle de ces théories, l'intermédialité est, d'un point de vue idéologique, une «question non questionnée», comme l'attestent deux exemples-clés pour moi :

5. Selon ces théories, il suffisait de quelques vocables pour inventer la matière d'un roman. Cette activité de «production » visait à bannir toute «illusion référentielle».

6. Christian Metz, Langage et cinéma, Paris, Éditions Larousse, coll. «Langue et langage », 1971, p. 179-180. 
- la focalisation selon Genette: après avoir affirmé dans Figures III que la focalisation interne suppose «en toute rigueur que le personnage focal ne soit jamais décrit, ni même désigné de l'extérieur7" ", Genette en trouve le meilleur exemple dans Rashômon (1950), le film d'Akira Kurosawa qui, malgré son parti pris de restriction cognitive, montre les personnages foyers de récit ${ }^{8}$;

- la métaphore récurrente chez les littéraires de «l'œil-caméra » pour désigner une description neutre et objective.

Si l'un de mes livres doit son titre à cette dernière expression, ce n'est pas pour entériner l'usage de la métaphore. Bien au contraire, c'est pour en éprouver la validité en la restituant à son contexte d'origine, le cinéma. De cette expérimentation, je conclus que le point de vue perceptif et le point de vue cognitif ne marchent pas de concert dans un média comme le cinéma qui utilise pourtant le point de vue comme un trait constitutif de langage. Et je montre, dans un second temps, que cette distinction conceptuelle s'impose aussi pour le roman, ce dont

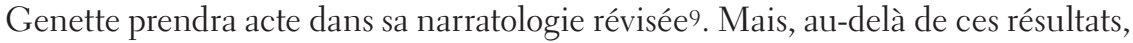
ce que je voudrais mettre ici en exergue, c'est la vertu heuristique de l'intermédialité qui guide ce mouvement de balancier entre film et roman. L'intermédialité perd ici sa dimension militante de mise à l'épreuve des potentialités expressives du matériau, pour servir le travail conceptuel, tel que le définit Georges Canguilhem dans une définition qui reste pour moi une maxime indépassable:

Travailler un concept, c'est en faire varier l'extension et la compréhension, le généraliser par l'incorporation de traits d'exception, l'exporter en dehors de sa région d'origine, le prendre comme modèle ou, inversement, lui chercher un modèle, bref lui conférer progressivement, par des transformations réglées, la fonction d'une forme ${ }^{10}$.

Cette logique de l'aller-retour consiste à comparer le fonctionnement d'un concept dans un média pour, ensuite, tester les résultats acquis dans un autre. Curieusement, l'intermédialité n'a pas tout à fait le même rôle selon qu'il s'agit,

7. Gérard Genette, Figures III, Paris, Éditions du Seuil, coll. «Poétique », 1972, p. 209.

8. Gérard Genette, Figures III, p. 207.

9. Gérard Genette, Nouveau discours du récit, Paris, Éditions du Seuil, coll. «Poétique», 1983 .

10. Cette citation figurait en exergue des Cahiers pour l'analyse, édités par le Cercle d'épistémologie de l'École normale supérieure à la fin des années 1960. Elle se trouve à l'origine dans Georges Canguilhem, «Dialectique et philosophie du non chez Bachelard», Revue internationale de philosophie, vol. XVII, nº 66, 1963, p. $45^{2}$. 
en l'occurrence, de le réimporter dans sa région d'origine ou de l'exporter. Pas le même rôle non plus, selon qu'on le définit en extension en compréhension ou qu'on le prend comme «modèle », comme l'atteste mon expérimentation sur le point de vue.

Ramener le concept sur le terrain du cinéma, c'est d'abord lutter contre l'obstacle épistémologique que représente, selon Gaston Bachelard, une métaphore inadéquate. C'est aussi forger un outil plus proche de la réalité qu'il pense et opérer une mise au point nécessaire. Tout cela ne me semble guère contestable. Les retombées du retour vers le média qui a vu naître la métaphore sont, en revanche, beaucoup plus imprévisibles. À quoi peut servir la distinction point de vue cognitif-point de vue perceptif, s'agissant d'un média qui n'est pas, comme le roman, intrinsèquement lié à l'œil, au regard et à l'oreille?

En fait, il s'avère, à y regarder de plus près, que la distinction conceptuelle existe aussi dans le roman, existence que l'analyse littéraire, cantonnée à son corpus d'œuvres écrites, n'a pu percevoir. L'exemple le plus frappant est encore celui de la focalisation. Après avoir affirmé dans Figures III, que les questions Qui parle? et Qui voit? sont absolument différentes, Genette finit par reconnaître, dans Nouveau discours du récit, que la focalisation est contrainte par la situation narrative :

[...] le narrateur homodiégétique est tenu de justifier (“comment le sais-tu?") les informations qu'il donne sur les scènes d'où il est absent comme personnage, sur les pensées d'autrui, etc., et toute information à cette charge fait paralepse [...]. On pourrait donc dire que le récit homodiégétique subit en conséquence une restriction modale a priori, et qui ne peut être évitée que par effraction ou contorsion perceptuelle. Pour désigner cette contrainte, faudrait-il parler de pré-focalisation ${ }^{11}$ ?

Pour Jaap Lintvelt, de même, la narration homodiégétique exclut le type neutre $^{12}$. En somme, le point de vue serait déterminé par la voix narrative, ce qui rendrait, en fin de compte, inutile la distinction voix-mode. Sans entrer dans les détails de mon argumentation, publié dans Texte et médialité13, j’oppose à Genette, grâce au détour par le cinéma, d’abord, que cette dissociation de la focalisation (point de vue cognitif) et de l'ocularisation (le point de vue oculaire)

11. Gérard Genette, Nouveau discours du récit, p. 53.

12. Jaap Lintvelt, Essai de typologie narrative. Le point de vue: théorie et analyse, Paris, Éditions José Corti, 1981, p. 79.

13. François Jost, «Propositions pour une narratologie comparée», dans Jürgen E. Müller (dir.), Texte et médialité, Mannheim, Lehrstuhl Romanistik I, Universität Mannheim, coll. «Mannheimer Analytika», 1987, p. 251-265. 
est la situation narrative la plus fréquente dans les films, quand un personnage raconte son histoire en flash-back, mais, surtout, les analyses de séquences filmiques me fournissent la preuve que le Je Narrant, la voix narratrice, n’en sait pas forcément autant que le Je Narré. Je prends l'exemple des Trois couronnes du matelot (1983), de Raoul Ruiz, dans lequel un personnage reçoit un coup de poing en ocularisation interne primaire et se retrouve en prison tandis qu'on l'entend commenter: "Je ne sais comment je me trouvais mêlé à la bagarre du côté des frères. Après, on m’a raconté qu'il y avait des morts et qu'on risquait une lourde peine. »

Plus genettien que Genette, je peux alors facilement mettre en évidence que l'auteur de Figures III a perdu de vue la distinction voix-mode qu'il avait introduite, pour n'avoir pas imaginé que cette dissociation du sujet, qui semblait intuitivement rare, était en fait très courante au cinéma. Ce qui, ipso facto, lui a fait négliger l'opérativité de cette distinction dans le domaine littéraire, dont témoigne la Vie d'Henry Brulard (1835), dans laquelle Stendhal revoit très bien son passé au point de dessiner le lieu où il se trouvait (ocularisation interne primaire: «je me vois et je vois Séraphie au point $\mathrm{S}$ »), tout en ne sachant plus très bien dans quelle disposition émotive il se trouvait (focalisation externe: «il me semble que je pleurais de rage $\left.{ }^{14} »\right)$.

J'insiste sur le fait que, pour avoir une valeur heuristique, l'intermédialité doit être un aller-retour et non un aller sans retour. J'en veux comme contreexemple la façon dont Jean-Marie Schæffer traite des différents médias. Dans un souci de cerner leur spécificité, il part d'une définition restrictive de la narration et du récit, qui les fait ressortir au seul domaine de l'écrit et de la lecture et qui, du même coup, dénie au film le statut de récit: «Dès lors [qu'une séquence] est filmée, elle se donne à voir et à entendre comme une représentation perceptivement accessible d'une séquence d'actions; dès lors qu'elle est racontée (au sens technique du terme), elle se donne à lire comme énoncée par un narrateur ${ }^{15}$. »

En raison de cette pétition de principe, exit la question de l'énonciation. «Le spectateur ne voit pas le film comme quelque chose que quelqu'un lui montrerait, mais comme un flux perceptif qui est le sien propre $^{16}$. Cette mise à la porte

14. Henri Beyle de Stendhal, Vie d'Henry Brulard [1835], dans Euvres intimes, Henri Martineau (éd.), Paris, Éditions Gallimard, coll. «Bibliothèque de la Pléiade », 1955 , p. 137.

15. Jean-Marie Schaeffer, Pourquoi la fiction?, Paris, Éditions du Seuil, coll. «Poétique», 1999, p. 304.

16. Jean-Marie Schaeffer, Pourquoi la fiction?, p. 301. 
du film du domaine de la narratologie ne se préoccupe guère, en l'occurrence, du fait que les spectateurs se racontent les films les uns aux autres, ce qui prouve la traductibilité de ce flux perceptif, et qu'ils vont d'abord au cinéma pour se faire raconter des histoires. Mais cette conception, surtout, reste très attachée à une esthétique qu'en l'occurrence Jean-Marie Schaeffer doit chérir, celle de la transparence, qui annule toute opération discursive au bénéfice du flux perceptif du spectateur.

La diversité des médias peut donc être pensée sur deux modes: l'un, qui est celui de Schæffer, que j’appellerai ségrégationniste, qui s'efforce de montrer que chaque média a une spécificité telle qu'il impose des concepts inexportables, et l'autre, celle que j'ai mise en œuvre sous le nom de narratologie comparée, que j'appellerai heuristique, puisqu'elle consiste à faire varier en extension et en compréhension des concepts peu ou prou universels ${ }^{17}$.

\section{MISE À L’ÉPREUVE DE LA THÉORIE PAR L'INTERMÉdIALITÉ}

On pourrait évidemment adresser à la conception de l'intermédialité que je viens de développer le reproche que Péguy faisait à Kant: non pas d'avoir les mains pures, mais de ne pas avoir de mains du tout. En effet, si l'aller-retour entre médiums a d'indéniables qualités pour travailler les concepts, les résultats restent fragiles tant qu'ils n'ont pas été confrontés à l'épreuve de la mise en contexte médiatique. J'ai souvent eu l'occasion de dire dans des colloques sur le cinéma et les autres arts que, parfois, certaines communications auraient aussi bien trouvé leur place dans un colloque sur le cinéma et les médias. Ce n'est pas que je sois personnellement détenteur d'une vérité révélée me permettant de faire le départ exact des activités qu'il convient de classer du côté de l'art ou du côté des médias. Si une telle exigence me paraît possible (et nécessaire), c'est au contraire que beaucoup de ces «pratiques culturelles » peuvent aussi bien ressortir à l'art qu'aux médias et que le seul critère pertinent, en l'occurrence, est le regard qu'on leur porte. En d'autres termes, aucune de ces pratiques n'est intrinsèquement artistique ou médiatique, tout dépend de l'approche de l'analyste. Du même coup, je crois assez peu à une approche neutre, qui ne choisirait pas son angle.

Prenons l'une de ces formules à l'emporte-pièce dont Godard a le secret: «Lumière, dernier peintre impressionniste». Son succès relatif, dont témoigne

17. André Gaudreault appartient de ce point de vue à la même démarche. Voir, entre autres, André Gaudreault, Du littéraire au filmique: système du récit, Paris, Québec, Méridiens Klincksieck, Presses de l'Université Laval, 1988 et André Gaudreault, François Jost, Le récit cinématographique, Paris, Éditions Nathan, 1990. 
en tout cas la façon dont Jacques Aumont la prend au sérieux dans L'œil interminable ${ }^{18}$, repose d'abord, bien sûr, sur la création d'une relation intermédiale insoupçonnée: le cinéma en tant qu'art mécanique n’est-il pas à l'opposé de la peinture, art libéral s'il en est, en cette fin du XIX ${ }^{\mathrm{e}}$ siècle?

Certes, il est légitime de trouver à ces pratiques maintes ressemblances: la distance, le cadrage ou le cadre, etc. Ces rapprochements, en l'occurrence, sous des couverts modernistes, ne font que prolonger la réflexion de Metz sur les codes à manifestations multiples, mais non universelles, en s'intéressant aux intersections de deux arts. Mais ce qu'on peut reprocher à ce type d'analyse, c'est qu'elle évite la question centrale du statut de ce que, faute de mieux, j’ai, jusqu'à présent, nommé «pratique culturelle». Or, de ce point de vue, la comparaison entre cinéma des débuts et peinture impose des réflexions beaucoup plus nuancées. En tant qu'art, le cinéma naissant - qui ne revendique d'ailleurs pas cette étiquette - emprunte beaucoup plus à la peinture d'atelier qu'à la peinture de son époque: le film est fabriqué dans l'atelier de pose et, comme la fresque du Moyen Âge, il sollicite toute une équipe où certains sont spécialistes des costumes, d'autres de décors, d'autres, enfin, de la direction d'acteurs. La consultation des catalogues et des journaux professionnels du début du $\mathrm{xx}^{\mathrm{e}}$ siècle nous apprend que celui qui fait le film, le réalisateur, n’a aucune existence propre; le cinéma est un art mécanique, un art de la main fait par des tourneurs de manivelle, dont on vend les produits au mètre, comme jadis les fresques ou les peintures d'atelier. En bref, le cinéma ne colle nullement à l'idée de l'art de son époque.

Regardons, à présent, le même objet - le cinéma des débuts — avec d'autres lunettes, des lunettes médiatiques. Contrairement au peintre du dimanche, dont personne ne contestera qu'il vise à faire de l'art, quelles que soient les qualités de sa production, Lumière n'avait nullement une telle visée en tête en tournant ses premières vues et la question de l'auteur n'a été soulevée par les éditeurs et les «écranistes » qu’à partir du moment où ils se sont posé des problèmes de répartition des droits, c'est-à-dire quand, précisément, le film a cessé d'être vendu au mètre pour être loué. Dès lors, la question de l'art a surgi non pas comme un simple débat philosophique, mais comme la pierre angulaire de la légitimation du cinéma par lui-même. Vu sous cet angle, le cinéma apparaît comme bien différent des autres arts, puisque c'est sans doute le premier (le seul) dont l'artisticité ait été plaidée grâce à la communication médiatique. Non seulement le statut d'art a été argumenté par les affiches, les catalogues, par l'excellence des

18. Voir Jacques Aumont, "Lumière, "le dernier peintre impressionniste" ", dans Loeil interminable: cinéma et peinture, Paris, Librairie Séguier, 1989, p. 13-36. 
œuvres adaptées bien plus que par celles de celui qu'on appellera le cinéaste, mais, en outre, les zélateurs de cette nouvelle activité de duplication ont compris très vite que le sort artistique du cinéma dépendait très largement des institutions communiquant le film au public: cinémathèques, critiques et salles. En bref, l'art cinématographique s'est construit sur la capacité du cinéma à devenir un média de masse (à la différence du kinétoscope d'Edison) et, en cela, je crois qu'il diffère de la plupart des arts qui l'ont précédé. Attentifs aux figures ou à la plasticité, les purs esthètes du cinéma commettent aujourd'hui la même erreur à propos du cinéma contemporain en réduisant l'œuvre à sa manifestation la plus improbable, le visionnement sur magnétoscope pour personne seule, négligeant l'extension visuelle qu'a fait subir à l'œuvre le DVD, avec sa cohorte de bonus, de making of et de péritextes en tout genre, destinés à mieux médiatiser l'œuvre.

Second exemple de cette nécessité d'aborder les faits culturels en les considérant sous l'angle médiatique Big Brother. Qu'est-ce qui fait que cette émission, qui se donne pour but explicite d'observer des gens jour et nuit, devient un succès mondial, alors que, quelques décennies auparavant, ce nom était seulement synonyme de totalitarisme, comme l'atteste Alphaville (Jean-Luc Godard, 1965)? Je ne prétends pas répondre en quelques lignes à cette question complexe, à laquelle j'ai récemment consacré un livre' ${ }^{19}$, mais je voudrais seulement souligner ici que l'explication de ce succès ne se trouve ni dans une révolution subite des mentalités ni dans l'histoire de la télévision, mais dans l'observation de nombreux phénomènes attestés par l'évolution des médias de diffusion comme le téléphone, l'ordinateur ou les caméras de surveillance.

De même que le «minitel rose », qui a fleuri en France dans les années 1980, institutionnalisait des conversations fortuites dues au mauvais état du réseau téléphonique de l'après-guerre, ce qu'on appelait le « réseau », le dispositif télévisuel de Big Brother a récupéré les tentatives individuelles et isolées de quelques jeunes femmes, comme la dénommée Jenni, qui braquaient au début des années 1990 une webcam sur leur chambre à coucher. Si l'on ajoute à cela le développement considérable des caméras de surveillance dans les lieux publics et l'argumentation sécuritaire qui a accompagné leur extension, on comprend que l'espionnage quotidien de la vie d'autrui a pu finalement apparaître à certains comme une activité normale.

Dans les deux exemples que je viens de prendre, passer d'un média à l'autre pour comprendre un phénomène n'a plus la même finalité que précédemment: il

19. François Jost, Lempire du loft, Paris, La Dispute éditeurs, coll. «Des mots sur les images ", 2002. 
s'agit moins d'affiner des concepts ou de varier les angles d'attaque que de déceler des symptômes d'un fait culturel qui prend soudain une ampleur insoupçonnée. Encore faut-il, pour que le concept d'intermédialité prenne pleinement son sens, que ce fait culturel soit d'ordre médiatique et que «média» ne soit pas un mot éponge qui absorbe et contienne indifféremment les questions d'art, d'adaptation ou de matière de l'expression (cf. Lumière et l'impressionnisme). Conçue dans cette perspective, l'épreuve de l'intermédialité est une étape obligée de l'histoire culturelle, comme le montre le cas de Big Brother. Le passage d'un média à l'autre devient une démarche heuristique nécessaire à la construction d'une relation intelligible entre des causes et des effets.

De l'intermédialité militante à la démarche raisonnée de l'historien, en passant par la mise à l'épreuve des concepts par le théoricien, l'intermédialité a de multiples usages. Si cet article vise à en esquisser la généalogie, il ne faudrait pas conclure pour autant que chaque étape rend caduque la précédente: elle l'englobe. Aussi ne me paraît-il pas exagérer de demander au chercheur d'aujourd'hui de s'interroger, à chacune de ses analyses d'un document, sur la pertinence de ce qu'il avance en le soumettant au crible de cette triple intermédialité. 